\title{
Comparative Review on SHS Thermodynamics Applied for Frontal Polymerization of Acrylamide and Triethylene Glycol Dimethacrylate
}

\author{
András JESZENSZKY, ${ }^{1}$ Sándor SZABÓ, ${ }^{2}$ and István P. NAGY ${ }^{1, \dagger}$ \\ ${ }^{1}$ Department of Physical Chemistry, University of Debrecen, P. O. Box 7, H-4010 Debrecen, Hungary \\ ${ }^{2}$ Department of Solid State Physics, University of Debrecen, P. O. Box 2, H-4010 Debrecen, Hungary
}

(Received June 16, 2005; Accepted November 28, 2005; Published April 15, 2006)

\begin{abstract}
The applicability of the thermodynamic considerations of Self-Heating High-Temperature Synthesis (SHS) for frontal polymerization of acrylamide and triethylene glycol dimethacrylate is reported. The basic concept of our work is based on certain analogies existing between frontal polymerization and SHS processes. The measurements involve the determination of the heat capacity of the starting materials, as well as the product's. The apparent activation energy of the reaction has also been studied. On the basis of the results, further new models are also set up. For instance, the role of convective flow in the low temperature initial phase might be supposed. [DOI 10.1295/polymj.38.364] KEY WORDS Self-Heating High-Temperature Synthesis / Frontal Polymerization / Thermodynamics / Apparent Activation Energy /
\end{abstract}

It is well-known that several solid-state chemical reactions are accompanied by the evolution of heat and the amount of this heat varies over a wide range of $100-500 \mathrm{~kJ} / \mathrm{mol}$. If such a reaction is sufficiently exothermic, a mixture of the powdered starting materials may be ignited by a hot thermal source (e.g. heated coil, laser beam). Upon initiation, from the point of ignition, a combustion wave is formed, self-propagating through the reactants, converting them into a product. ${ }^{1}$ This method, also referred to as combustion synthesis, does not require any additional heat since the reaction becomes self-sustaining after a short time. It is mainly used in the manufacture of certain so-called advanced materials. These include over 300 compounds such as various nitrides ( $\mathrm{ZrN}, \mathrm{TaN}, \mathrm{HfN})$, borides $\left(\mathrm{TiB}_{2}, \mathrm{ZrB}_{2}\right.$, $\left.\mathrm{HfB}_{2}\right)$, carbides $(\mathrm{TaC}, \mathrm{WC}, \mathrm{SiC})$, silicides $\left(\mathrm{MoSi}_{2}\right.$, $\mathrm{Ti}_{5} \mathrm{Si}_{3}$ ), composites ( $\mathrm{TiC}-\mathrm{TiB}_{2}, \mathrm{~B}_{4} \mathrm{C}-\mathrm{Al}_{2} \mathrm{O}_{3}$ ), intermetallics (NiAl, $\mathrm{NbGe})$ and also superconductors $\left(\mathrm{YBa}_{2}-\right.$ $\mathrm{Cu}_{3} \mathrm{O}_{7-x}$ ). Combustion synthesis can be conducted in two ways, the self-propagating high-temperature synthesis (SHS) mode and the thermal explosion mode. The principal difference between these two modes is that in the case of SHS the reaction is initiated at one end of the sample, while thermal explosion is initiated by heating the whole sample uniformly.

As far as the analogies between SHS and frontal polymerization are concerned, consider the temperature and conversion profiles, keeping in mind that the range of the temperature of frontal polymerization is much narrower $\left(250-300^{\circ} \mathrm{C}\right)$ than that of SHS processes $\left(1000-2000^{\circ} \mathrm{C}\right)$. If these temperature ranges, however, are divided by the temperature of reactions, then the two regions are similar to each oth- er. Figure 1 depicts the temperature and conversion profile types mentioned above.

Here we give a short survey of the thermodynamic considerations reported in the paper by Holt and Dunmead (1991). ${ }^{3}$ Consider a solid-state SHS reaction in which $M$ and $X$ (both are solids) react with each other to give the product $M X$.

$$
M_{(\mathrm{s})}+X_{(\mathrm{s})} \rightarrow M X_{(\mathrm{s})}
$$

An important parameter in this context is the adiabatic temperature $\left(T_{\mathrm{ad}}\right)$ which stands for the temperature achieved during adiabatic conditions and complete conversion of the starting materials into product. Under these circumstances, according to the conservation of energy, the enthalpy of the starting materials at the initial temperature $\left(T_{\text {in }}\right)$ equals that of the product at the adiabatic temperature. The difference of these pa-

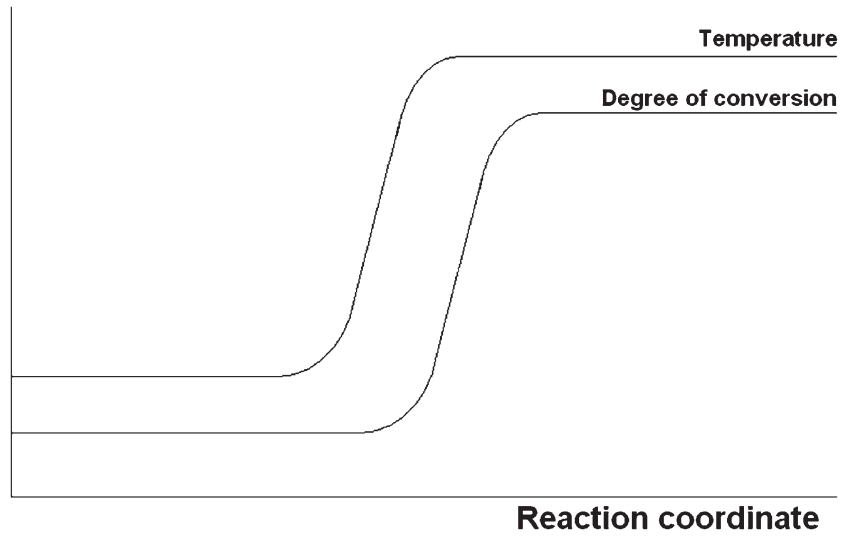

Figure 1. Temperature and conversion profiles of SHS and frontal polymerization.

${ }^{\dagger}$ To whom correspondence should be addressed (Tel: +36-52-512-900/2386, E-mail: 3586nagy@tigris.klte.hu). 
rameters, in fact, is equal to the reaction heat, that is:

$$
H\left(T_{\text {ad }}\right)_{M X}-H\left(T_{\text {in }}\right)_{M X}=Q
$$

In case of initiation under standard conditions, the heat of reaction of course would equal the negative of the standard heat of formation of the product $M X$. The initiation, however, takes place at a higher temperature, thus we can write:

$$
\begin{aligned}
& Q=\left(-\Delta H_{\mathrm{f}, 298}\right)-\int_{298}^{T_{\mathrm{in}}} \Delta C_{p} d T \\
& Q=\int_{T_{\text {in }}}^{T_{\mathrm{ad}}} C_{p}(M X) d T
\end{aligned}
$$

where $\Delta C_{p}$ stands for the difference between the specific heat of the product and those of the reactants at constant pressure. As it is known, several compounds obey the Neumann-Kopp rule, that is the difference of the specific heats is negligible, $\Delta C_{p} \approx 0$. In this case, (3) simplifies to:

$$
\int_{T_{\mathrm{in}}}^{T_{\mathrm{ad}}} C_{p}(M X) d T=-\Delta H_{\mathrm{f}, 298}
$$

This equation is only valid when the product is a single solid phase. In case of partial or total melting, or any other solid-solid transformations, we can consider these phenomena as well, that is:

$$
\begin{aligned}
\left(-\Delta H_{\mathrm{f}, 298}\right)= & \int_{T_{298}}^{T_{\mathrm{t}}} C_{p}(\alpha) d T+\Delta H_{\mathrm{t}}+\int_{T_{\mathrm{t}}}^{T_{\mathrm{m}}} C_{p}(\beta) d T \\
& +\Delta H_{\mathrm{m}}+\int_{T_{\mathrm{m}}}^{T_{\mathrm{ad}}} C_{p}(\text { liq }) d T
\end{aligned}
$$

where $\alpha$ and $\beta$ represent two solid phases, and $T_{\mathrm{m}}$ is the melting point of phase $\beta$.

Without going into details, the readers are referred to the paper of A. Varma and JP. Lebrat ${ }^{3}$ as far as the determination of the activation energy is concerned. We just give the final equation, which can be calculated from the macroscopic energy and mass balance equations. For one-dimensional propagation we arrive at the following result:

$$
v^{2}=\frac{\lambda}{\left(-\Delta H_{\mathrm{f}}\right) \rho} \frac{R T_{\mathrm{c}}^{2}}{E} \frac{k_{0} \exp \left(-\frac{E}{R T_{\mathrm{c}}}\right)}{f\left(\eta_{\mathrm{s}}\right)}
$$

where $v$ is the velocity of propagation, $\lambda$ the thermal conductivity, $\Delta H_{\mathrm{f}}$ the heat of formation, $\rho$ the density, $R$ the universal gas constant, $T_{\mathrm{c}}$ the temperature of combustion, $E$ the activation energy, $k_{0}$ the pre-exponential factor, $f\left(\eta_{\mathrm{s}}\right)$ is the function of conversion. It can be seen from (7) that the plot of $\ln \left(v / T_{\mathrm{c}}\right) v s$. $1 / T_{\mathrm{c}}$ provides a straight line with a slope proportional to the apparent activation energy. This equation is val- id for transformations for which the rate of reaction is controlled by the temperature of the reaction front.

\section{EXPERIMENTAL SECTION}

\section{Chemicals and Experimental Methods}

Acrylamide (AA, Reanal, particle size: $30-50 \mu \mathrm{m}$ ), triethylene glycol dimethacrylate (TGDMA, Aldrich, 95\%), 2,2'-azoisobutyronitrile (AIBN, Fluka, >98\%, particle size: $30-50 \mu \mathrm{m}$ ) were reagent grade.

Acrylamide was ground in a rock tumbler with ceramic beads for about $8 \mathrm{~h}$ until a uniformly powdered mixture was obtained, then AIBN was mixed in with a mortar and pestle. TGDMA was finally added and mixed carefully. The highly viscous mixture was packed into $9 \mathrm{~mm}$ (i.d.) glass tubes and polymerization fronts were initiated with a soldering iron. A region of solid polymer could be observed to propagate through the highly viscous monomer mixture. No noticeable melt zone was observed.

\section{Investigation of the Apparent Activation Energy}

The front velocities were determined at various temperatures ranging from 20 to $80^{\circ} \mathrm{C}$ by following the movement of the front (which is perfectly perceptable to the eye) by $30 \mathrm{~s}$. The velocities were determined from the slopes of distance-time graphs. The required temperature was achieved by external thermostation of the glass tube, in which the frontal polymerization was carried out. This system consists of a vacuum pump and a thermostate, regulating the ambient temperature around the glass tube.

\section{Determination of the Heat and Heat Capacity}

The standard heat of formation (the heat of reaction under standard conditions) evolved during frontal polymerization was measured by calorimetry. It was determined at various monomer ratios (ranging from 0 to 1 molar ratios), by measuring the temperature of the water in a nearly adiabatic system consisting of the reacting system in the glass tube and an external vessel.

Perkin Elmer DSC 7 instrument was used for measuring the heat of reaction at the temperature of the reaction front. The reaction was investigated at different heating rates from 10 to $500 \mathrm{~K} / \mathrm{min}$. Measurements were performed in air or under He (purity 4.6) atmosphere. Specific heats of the monomers and that of the product were also determined by differential scanning calorimetry and the measurements provided the heat capacities directly.

\section{RESULTS AND DISCUSSION}

The behaviour of the activation energy and the value of apparent activation energy were studied and de- 
termined from (7) and (11). We measured the velocity of the propagating polymerization front at various temperatures and in every case linear behaviour was observed. Velocity was constant as the function of time and covered a range of $0.018-0.035 \mathrm{~cm} / \mathrm{s}$. The error of the measurements may be relatively large concerning the value of apparent activation energy due to the narrow temperature and time range in which the polymerization process was studied. However, the linear behaviour of the $\ln \left(v / T_{\mathrm{c}}\right) v s .1 / T_{\mathrm{c}}$ graph seems to be true and the magnitude of the apparent activation energy is also acceptable for this kind of process.

At first, applicability of (7) was investigated. In the SHS model (J. B. Holt, S. D. Dunmead 1991) ${ }^{3}$ adiabatic transformation is supposed, and $T_{\mathrm{c}}$ in (7) is the temperature of the reaction front at the final state. Considering the adiabatic conditions, this temperature depends in a linear fashion on the temperature of thermostation (the temperature of the initial components). The real transformation is, of course, not fully adiabatic, but in accordance with (7), the plot $\ln \left(v / T_{\mathrm{c}}\right) v s .1 / T_{\mathrm{c}}$ can be well assumed as a straight line (Figure 2).

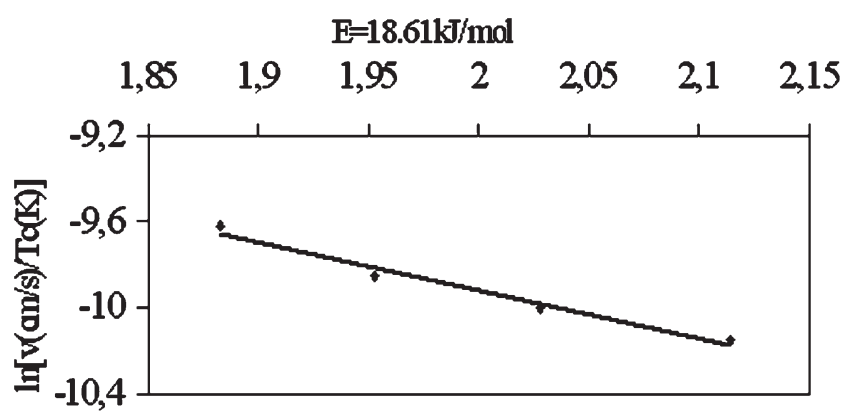

1/[Tc(K)E-3]

(a)

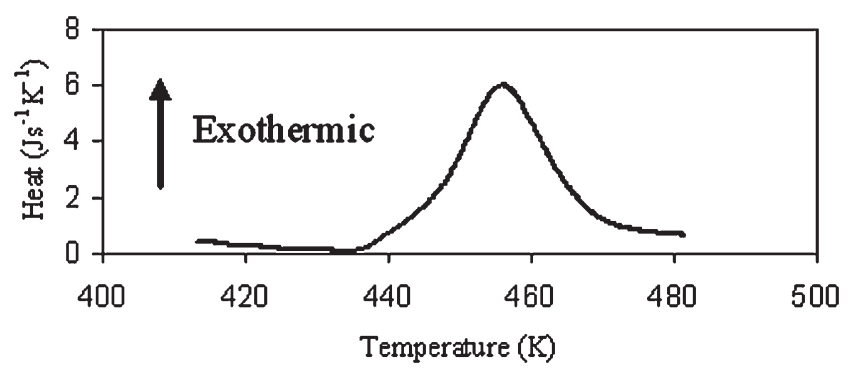

(b)

Figure 2. Investigation of the activation energy on the basis of (7). (a): plot $\ln \left(v / T_{\mathrm{c}}\right) v s .1 / T_{\mathrm{c}}$ can be assumed as a straight line. (b): DSC curve for the approximation of $T_{\mathrm{c}}$ (heating rate is $50 \mathrm{~K} /$ $\min ) . T_{\mathrm{c}}$ is approximated with $473 \mathrm{~K}$ in the conditions for which the thermostation temperature of the initial components was the ambient temperature. Due to adiabatic conditions, we supposed that the increase in $T_{\mathrm{c}}$ is the same as that one of the thermostation of the initial components.

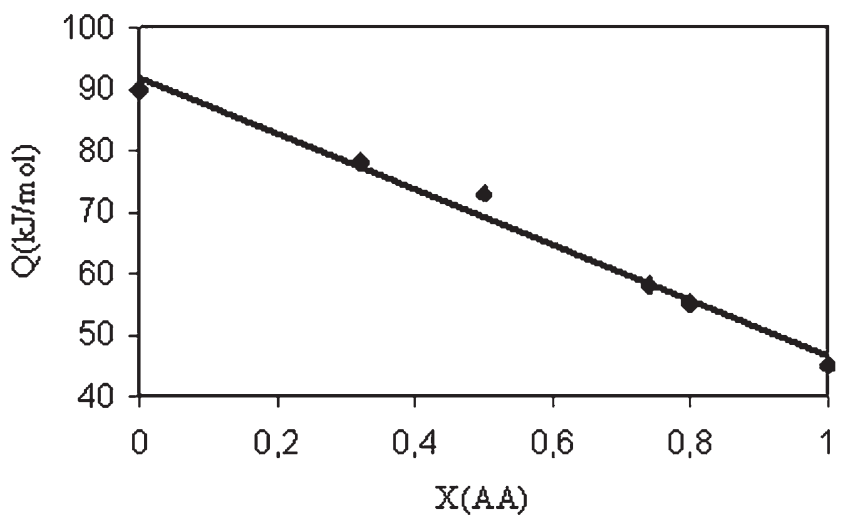

Figure 3. Determination of the heat of reaction of the formation of one mole copolymer at $x_{\mathrm{AA}}=0.5$. The value of the heat of reaction $\left(-\Delta H_{\mathrm{f}, 298}\right)$ at 0.5 molar ratio is $73 \mathrm{~kJ} \mathrm{~mol}^{-1}$.

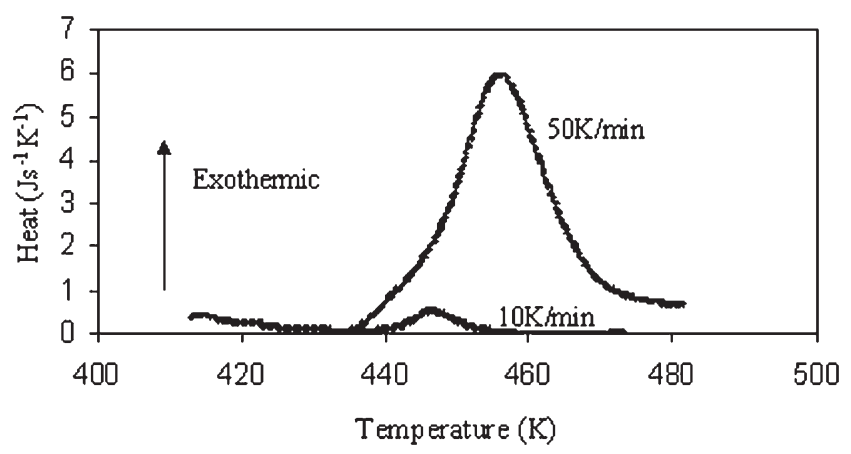

Figure 4. DSC peaks of AA + TGDMA $\rightarrow$ AATGDMA copolymerization reaction with different heating rates. With real $50 \mathrm{~K} / \mathrm{min}$ rates the heat of transformation (Q) was found to be $(43.4 \pm 5.7) \mathrm{kJ} \mathrm{mol}^{-1}$. Due to the effective decay of the components this heat was much less if the heating rate was substantially lower or larger than in the case of normal frontal copolymerization. Antecendent reactions of the components before copolymerization can also effectively depress the transformation peak.

Applicability of the SHS model was also investigated on the bases of (1)-(5). The standard heat of formation $\left(\Delta H_{\mathrm{f}, 298}\right)$ was determined at various molar ratios of AA and TGDMA from 0 to 1 . The calculations provided the heat of formation of one mole of copolymer. Figure 3 represents the values measured.

The heat of reaction at the $T_{\mathrm{c}}$ front temperature of the copolymer was also needed and it was measured by DSC (Figure $2 b$ and Figure 4 ).

Polymers decay above $373 \mathrm{~K}$ and the amount of the material dissolved depends on the heating rate. While during the heating processes with slow $(10 \mathrm{~K} / \mathrm{min})$ and fast $(500 \mathrm{~K} / \mathrm{min})$ heating rates the desintegration and evaporation were very effective, the decay was more moderated at 50 and $100 \mathrm{~K} / \mathrm{min}$. This interval $(50-100 \mathrm{~K} / \mathrm{min})$ is the real heating rate region in the case of frontal polymerization of the monomers. In accordance with the facts mentioned above, we obtained different reaction heats by different heating 
conditions. If the heating rates were in the real inter$\mathrm{val}$, the weight loss due to evaporation and decay was about $5 \mathrm{wt} \%$ and the heat of reaction $(43.4 \pm$ 5.7) $\mathrm{kJ} \mathrm{mol}^{-1}$.

The DSC measurements have also provided the temperatue-dependence of the molar heat capacity. The difference of the heat capacities of the copolymer and the initial monomer mixture $\left(\Delta C_{p}=C_{\mathrm{AATGDMA}}-\right.$ $C_{\mathrm{AA}+\mathrm{TGDMA}}$ ) can be approximated by a constant value of $-83 \mathrm{~J} \mathrm{~mol}^{-1} \mathrm{~K}^{-1}$. However, due to the decay and preliminary reactions of the components, the error of this value can be relatively large $\left( \pm 37 \mathrm{~J} \mathrm{~mol}^{-1} \mathrm{~K}^{-1}\right)$. Substituting the aforementioned molar heats and molar heat capacities in (3), and estimating $T_{\text {in }}$ as $435 \mathrm{~K}$ (on the basis of Figure 2b) we got:

$$
\begin{gathered}
Q=\left(-\Delta H_{\mathrm{f}, 298}\right)-\int_{298}^{T_{\text {in }}} \Delta C_{p} d T \\
43.4 \mathrm{~kJ} \mathrm{~mol}^{-1}=73 \mathrm{~kJ} \mathrm{~mol}^{-1} \\
-(-0.083 \cdot 137) \mathrm{kJ} \mathrm{mol}^{-1} \\
43.4 \mathrm{~kJ} \mathrm{~mol}^{-1}=84.4 \mathrm{~kJ} \mathrm{~mol}^{-1}
\end{gathered}
$$

It should be noticed that the left and right sides in (10), based on the SHS model, are in the same order of magnitude but they differ by a factor of 2 . The reason for this difference can be the decay and antecendent reactions of monomers before copolymerization. This can cook accounts and these processes must be taken into account besides the routinely used SHS model. It is also important to mention that the linear shape of the curve in Figure 2a is in good agreement with the SHS model but the activation energy $(18.61 \mathrm{~kJ} / \mathrm{mol})$ is much less than usual $(100$ $500 \mathrm{~kJ} / \mathrm{mol}$ ). Whereas this problem is eliminated if, on the basis of basic dimension analytical considerations (B. S. Massey, 1971), ${ }^{2}$ we consider that not the activation energies but the energies divided by transformation temperature ( $E_{\text {act }} / T_{\text {trans }}$ or $\left.E_{\text {act }} / k T_{\text {trans }}\right)$ must be in the same order of magnitude. This requirement is real because the transformation temperature of frontal polymerization is fifth-tenth part of that which occurs during the routinely used SHS reaction and model.

It is clear that SHS model can describe the frontal polymerization though the calculated energy values are correct only within an order of magnitude. The reason for this fact can be the decay and antecendent reactions of monomers before copolymerization. However, other possibilities and problems are also open which arise from the differences between SHS and frontal polymerization.

In the case of frontal polymerization a wide liquid region through a strong temperature gradient can exist before the reaction zone. It is a rational presumption then that the convective flow of unreacted liquid

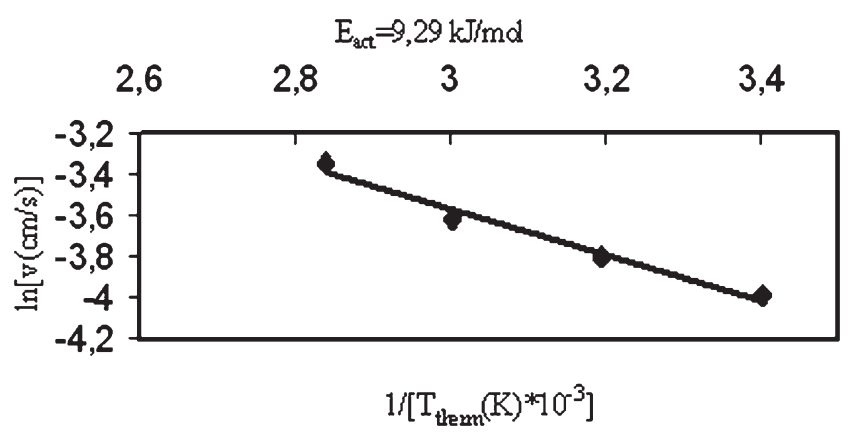

Figure 5. $\ln v v s .1 / T_{\text {therm }}$ in the case of frontal copolymerization of AA-TGDMA. The linear shape might confirm a model in which the convective flow of the liquid phase initial monomer mixture can have an important role in the rate of reaction and the velocity of the polymerization front.

monomers can also be determinative in the rate of copolymerization and in the rate of the reaction front. In this case the velocity of the transformation front might be determined by a simpler form:

$$
v \approx \frac{1}{\eta} \approx C_{1} \exp \left(\frac{-E}{R T_{\text {therm }}}\right)
$$

In (11) $C_{1}$ is a constant, $T_{\text {therm }}$ is the temperature of thermostation, $\eta$ is the viscosity of convective flow and $E$ is the activation energy in accordance to the Eyring-Andrade formula. In this case, $\ln v$ depends linearly on $1 / T_{\text {therm. }}$. From Figure 5 it is apparent that this linear behaviour is actually also valid within the experimental error.

In addition, it is well-known from the literature that a reaction front propagating linearly can exist in several cases if a fast and a subsequent slow process determine the reaction. For example, this happens in case of extremely strong assymmetry of the diffusion constants or subsequent fast diffusion and slow reaction may cause this kind of behaviour (Z. Erdélyi 2002, King-Ning Tu et al. 1992). This suggests that beside the SHS model other possible and refined conceptions may be taken into consideration.

\section{CONCLUSIONS}

The applicability of thermodynamic considerations of Self-Heating High-Temperature Synthesis (SHS) for frontal polymerization of acrylamide and triethylene glycol dimethacrylate was investigated. It have been found that the routinely used SHS model can well describe the processes but the calculated values are correct only within an order of magnitude. Decay and antecendents of the monomers and the copolymer must be taken into account in a more sophisticated model. Consideration of other models can also occur. For instance, the role of convective flow in the low 
temperature initial phase might be supposed. Our study also reveals that more complex systems can also be studied by the considerations applied for SHS and simpler approaches (see eqs (10) and (11)) may also be used.

Acknowledgment. The authors thank the financial support of the Hungarian Scientific Foundation (OTKA) grant No. T038078 and No. T038079.

\section{REFERENCES}

1. J. P. Lebrat and A. Varma, Chem. Eng. Sci., 47, 2179 (1992).

2. B. S. Massey, "Units, Dimensional Analysis and Physical Similarity," Van Nostrand-Reinhold, Princeton, New Jersey, 1971.

3. J. B. Holt and S. D. Dunmead, Annu. Rev. Mater. Sci., 21, 305 (1991). 